\title{
Haemoglobin and albumin as predictors of length of stay of spinal injured patients in a rehabilitation centre
}

\author{
R G Burr MSc PhD C Chem FRSC,${ }^{1}$ L Clift-Peace BSc SRD ${ }^{2}$ I Nuseibeh MB ChB \\ LMSSA FRCS(Ed) ${ }^{1}$ \\ ${ }^{1}$ National Spinal Injuries Centre and ${ }^{2}$ Department of Nutrition and Dietetics, Stoke \\ Mandeville Hospital, Mandeville Road, Aylesbury, Buckinghamshire, HP21 8AL, UK.
}

\begin{abstract}
The aim of this work was to identify factors related to the length of stay of patients admitted to this centre for rehabilitation. The study involved 200 patients admitted within one year of onset of a spinal cord lesion. Clinical and laboratory data were recorded. Length of stay was related to the level of the spinal cord lesion and whether it was complete or incomplete. The length of stay was increased in patients in whom anaemia or hypoalbuminaemia occurred; the cause of these conditions was not always evident. We conclude that attention directed towards recognising and treating the cause of anaemia or hypoalbuminaemia may be expected to shorten the time required for rehabilitation in this centre.
\end{abstract}

Keywords: paraplegia; spinal cord injury; anaemia; hypoproteinaemia; rehabilitation.

\section{Introduction}

Impaired nutritional status leads to increased morbidity and mortality and prolonged stay in hospital among surgical and geriatric patients. ${ }^{1.2}$ A substantial proportion of spinal cord injured patients are reported to be malnourished. ${ }^{3-6}$ Low serum albumin, viewed as a nutritional indicator, correlates well with delayed completion of rehabilitation and reduced mobility outcome. $^{7}$

Plasma proteins, however, are greatly influenced by stress and sepsis and are now considered indices of severity of illness rather than of nutritional state. ${ }^{8}$ Other commonly used anthropometric and laboratory indicators of nutritional status, such as skinfold thickness and plasma proteins, lack reproducibility and specificity. ${ }^{9.10}$ Alterations in the metabolic rate following trauma or paralysis render dietary assessment difficult. ${ }^{11,12}$

Prolonged stay in hospital is costly. ${ }^{13}$ It is desirable to identify responsible factors. If these factors can be identified, management directed at modifying them may speed the process of rehabilitation.

\section{Patients and methods}

The study involved 200 patients admitted within one year of onset of a nonprogressive spinal cord lesion for management, treatment and rehabilitation. Patients under the age of 16 years, those who were discharged before completion of rehabilitation or who died within one year of onset of the cord lesion were excluded, as was a patient with the nephrotic syndrome. There were 155 male and 45 female patients, aged from 16-77 (median 30) years, 89 with lesions of the cervical, 88 of the thoracic and 23 of the lumbar cord, 107 being complete and 93 incomplete lesions. In 35 the pathology was nontraumatic in origin. Among those with traumatic lesions 50 had significant associated injuries, and 47 had internal fixation of the spine. The duration of the cord lesion on admission to this centre was from the day of injury to 11 months (median 26 days) after injury. The length of stay was from $1.5-14$ (median 5) months.

Blood haemoglobin $(\mathrm{Hb})$ and full blood count and serum albumin concentration (SA) were measured routinely for clinical management. Anaemia was considered to 
be present when the $\mathrm{Hb}$ was below $13.5 \mathrm{~g} /$ $\mathrm{dl}$ for a man and below $11.5 \mathrm{~g} / \mathrm{dl}$ for a woman. ${ }^{14}$ Where primary anaemia was diagnosed, appropriate clinical management was instituted. Mean cell volume $(\mathrm{mcv})$ in the range 85-95 was considered normocytic, in the range 70-80 slightly microcytic and $<70$ severely microcytic. Mean cell haemoglobin concentration (mchc) in the range $30-35 \mathrm{~g} / \mathrm{dl}$ was considered normochromic, in the range 26-30 slightly microchromic and $<26$ severely microchromic. Hypoalbuminaemia was considered to be present when the SA was below $35 \mathrm{~g} / \mathrm{l}$.

All patients who were clinically malnourished, whose nutritional intake was deemed to be inadequate or whose SA was low, received regular visits by a member of the department of nutrition and dietetics who gave active advice throughout. Parenteral or nasogastric tube feeding was administered where clinically indicated. The target intakes were $2000 \mathrm{kcal}$ including $100 \mathrm{~g}$ protein per day $(3000 \mathrm{kcal}$ including $150 \mathrm{~g}$ protein for acute lesions until the medical condition settled and the patient was able to take food orally).

The occurrence of pressure sores, respiratory complications and pyrexia for any cause were recorded. The occurrence of any of these was taken to be evidence of the presence of sepsis.

Statistical analysis of the data was by multiple regression analysis and multifactorial analysis of variance.

\section{Results}

Anaemia was observed in 98 patients. It was normocytic, normochromic in 48; normocytic and slightly microchromic in 32 ; slightly microcytic and slightly microchromic in 16 ; slightly microcytic with severe microchromia in 5; and severely microcytic with severe microchromia in 1 . Leucocytosis was present in 25 patients. The anaemia was associated with respiratory complications in 24 patients; with pressure sores in 21 ; with both in 7 ; with urinary tract infection in 7 ; with orchitis in 3 ; with abscess in 1 ; and with pyrexia of other causes in 5 . There was internal haemorrhage in 12 and one each had deep vein thrombosis, pul- monary embolus and malignancy. There were 2 patients with clotting mechanism defects and 2 with haemoglobinopathies. No cause for anaemia could be found in 14 patients.

Of 53 patients admitted within 7 days of spinal cord injury, 49 had decreased SA in the first 10 days following the trauma. Nine patients had decreased SA for 10 days or less following surgery. Seventy-four patients had reduced SA in the presence of respiratory complications (18), pressure sores (15), both (5), urinary tract infection (5), abscess (2), orchitis (1), other pyrexias (8) or malignancy (1). In 19 patients no cause of hypoalbuminaemia could be identified.

Nutritional supplementation was given to $65(33 \%)$ patients for $1-46$ (median 4$)$ weeks. A further 5 patients received dietetic advice for weight reduction (2), diabetes (2) or being a vegetarian (1).

The total number of patients with clinical evidence of malnutrition was $9(4.7 \%)$. This was present on admission in 6 , one being an epileptic lady who was very thin prior to her cord injury: the others were admitted 21-210 days after injury. Three showed a decline in clinical nutritional state after admission, which improved with vigorous nutritional support. The features associated with clinically poor nutritional state were: large or multiple infected pressure sores (5), infection of wounds and chest (1), depression (1), facial palsy and acute depression (1), head injury and multiple fractures (1).

In 24 patients who received nutritional support SA remained normal and there were no clinical signs of nutritional deficiency.

Length of stay was longer in patients with pressure sores or sepsis than in those without $(p<0.05$ and 0.0001 respectively). Anaemia was more common in patients with pressure sores than in those without $(p<0.05)$. Anaemia and hypoalbuminaemia were more common in patients with sepsis than in those without $(p<0.0001)$. However, multifactorial analysis of variance showed that the only significant correlates of length of stay were: level of cord lesion, completeness of cord lesion, the occurrence of anaemia and the occurrence of hypoalbuminaemia (Figs 1-5). Length of stay 


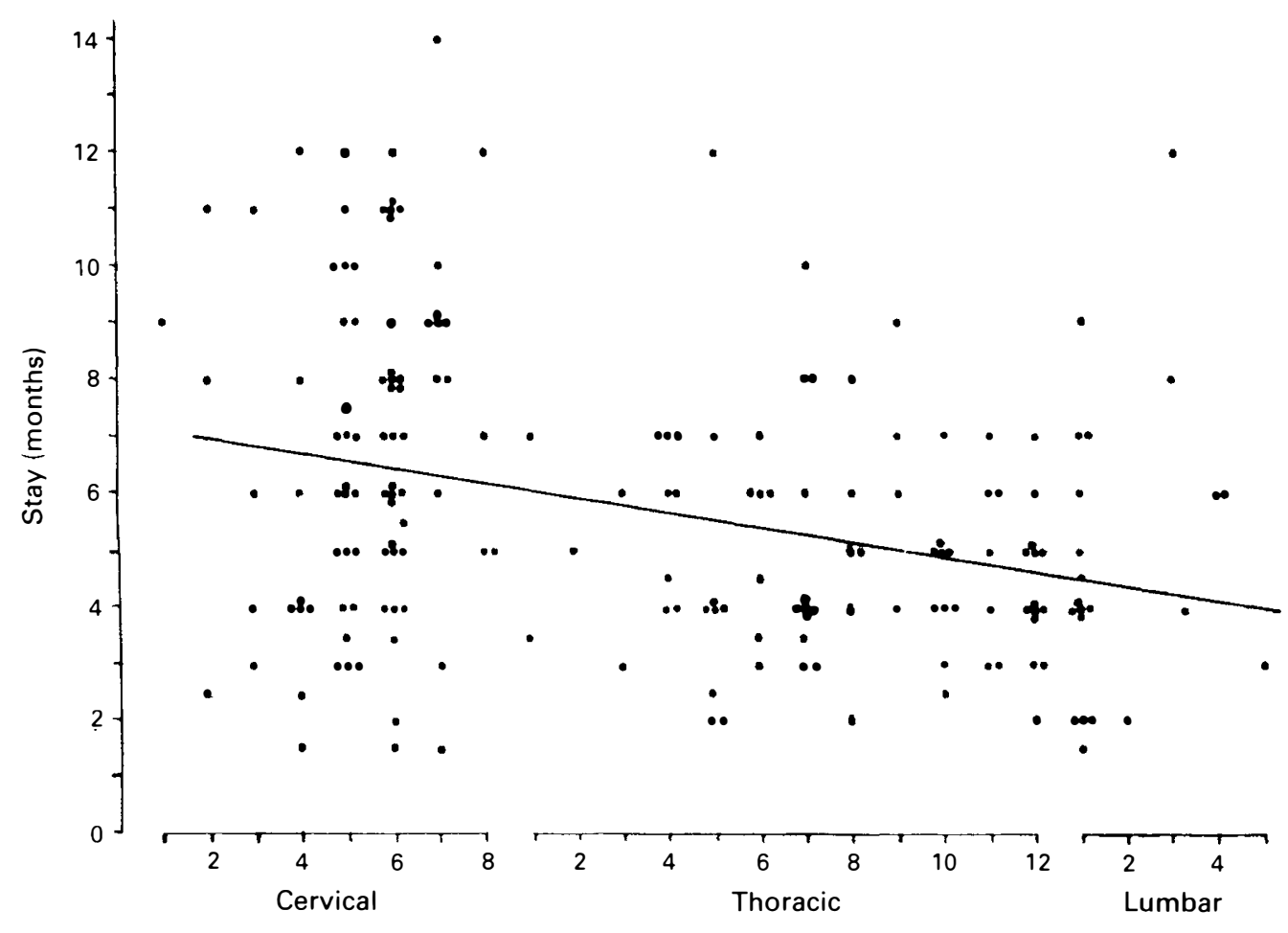

Neurological level of cord lesion

Figure 1 Length of stay in relation to level of cord lesion.

was not related to age, gender, traumatic origin of cord lesion, the occurrence of associated injuries, internal spinal fixation, clinical undernutrition or delay before admission.

\section{Discussion}

A more severe degree of paralysis, evidenced by a higher level of spinal cord lesion or a complete lesion, was associated with longer stay in hospital than in patients with lower or incomplete lesions (Figs 1, $2)$. For level of lesion this was expected. Patients with incomplete lesions, who frequently require more time and effort to realise their rehabilitation potential, were expected to stay longer than complete lesions, but they did not. The occurrence of anaemia or hypoalbuminaemia was also associated with prolonged stay (Figs 3,4).

A ready explanation for the relation between anaemia and prolonged stay might be that anaemia is associated with pressure sores which, because of the time necessarily spent in bed, will delay rehabilitation. However, while there was a significant correlation between the occurrence of anaemia and the occurrence of sores and also between the occurrence of sores and length of stay, there was a greater correlation between the occurrence of anaemia and length of stay. The same was true of hypoalbuminaemia. Consequently, when anaemia and hypoalbuminaemia were included as independent variables in the analysis of variance, the effects of sores and of clinical sepsis receded into statistical insignificance.

What is interesting therefore is that anaemia and hypoalbuminaemia increase length of stay even when not related to some obvious clinical cause of delayed rehabilitation such as a pressure sore or pyrexial illness. 


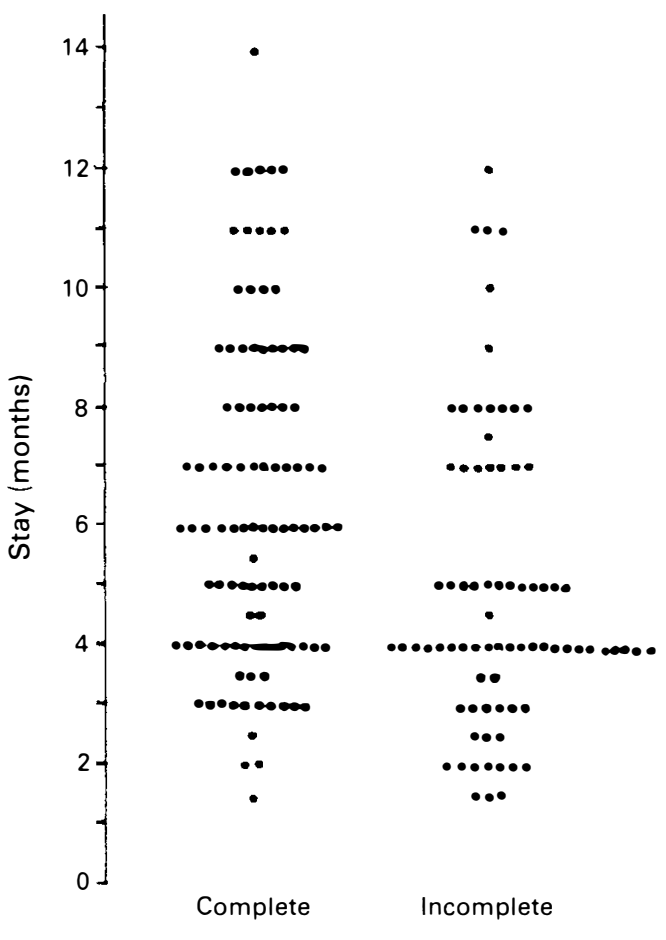

Cord lesion

Figure 2 Length of stay in patients with complete and incomplete cord lesions.

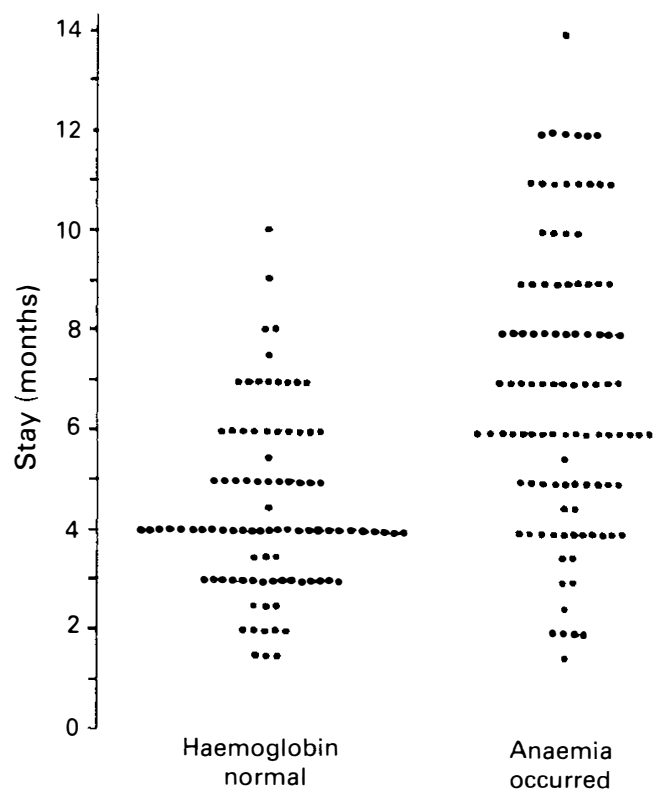

Figure 3 Length of stay in patients with and without anaemia.

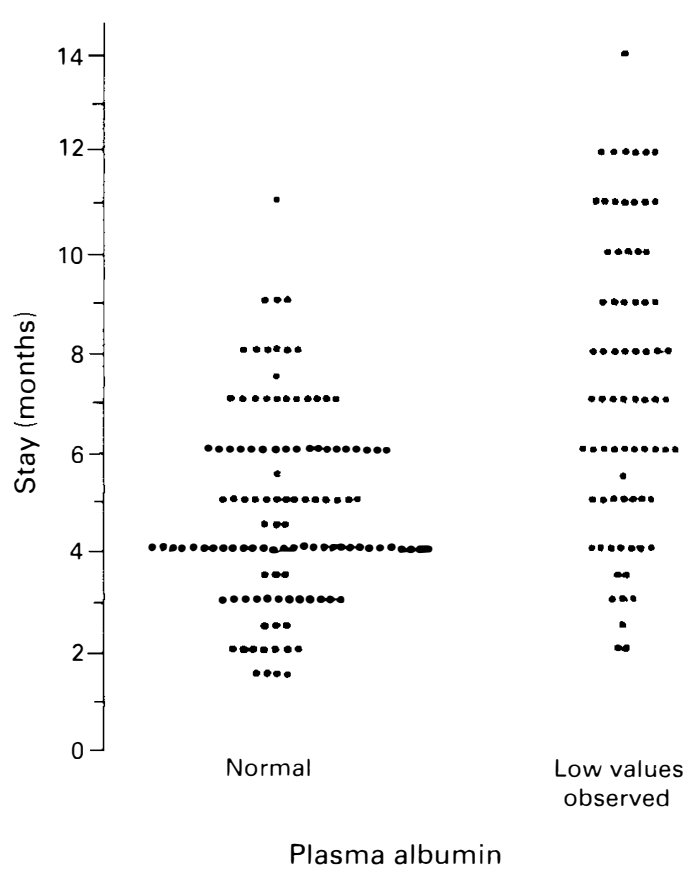

Figure 4 Length of stay in patients with and without hypoalbuminaemia.

In an appreciable number of patients with anaemia or hypoalbuminaemia, no clinical cause was apparent. Asymptomatic urinary tract infections were common and may have been partly responsible. We infer that the increased length of stay in hospital was caused either by unrecognised sepsis or by the debilitating effects of the anaemia and/ or hypoalbuminaemia. Length of stay in hospital depends on many factors-clinical, psychological, social, etc. This study indicates, however, that attention directed towards recognition and treatment of the factors responsible for anaemia and hypoalbuminaemia may be expected to speed up rehabilitation and shorten hospital stay.

Stay was not prolonged in patients showing clinical evidence of malnutrition. This may have been because of the small number of such patients $(4.7 \%)$ or because of the larger effect of other factors. Malnutrition may have contributed to the otherwise unexplained hypoalbuminaemia in some of our patients. Previous studies that have shown an effect of malnutrition on morbidity and length of stay have relied, in part at 


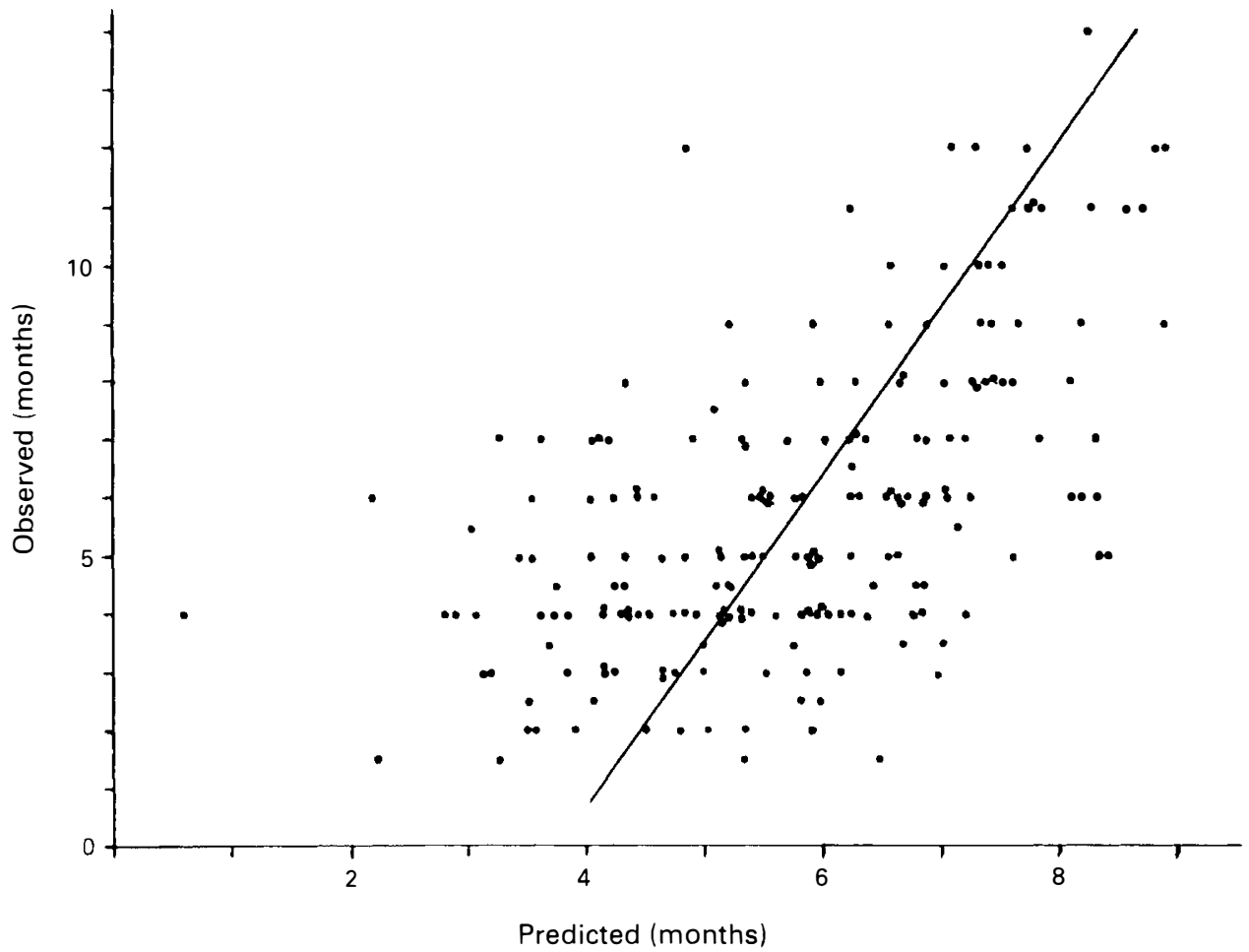

Figure 5 Observed and predicted length of stay. Predicted length of stay $=12.10470-0.09531$. Vert -0.83506 . Comp $+0.97686 . \mathrm{Hb}-0.16218$.Alb. Vert $=$ vertebral level of neurological cord transection numbered consecutively from $\mathrm{C} 1$. Complete lesion: Comp $=1$; incomplete lesion: Comp $=2$. Anaemia: $\mathrm{Hb}=2$; no anaemia: $\mathrm{Hb}=1$. Alb $=$ lowest serum albumin level more than 10 days following trauma or surgery. $\mathrm{R}$ squared (adjusted) $=0.3456$.

least, on plasma albumin as a nutritional indicator. Since this is now considered to be largely an index of severity of illness it is possible that these earlier studies should be reevaluated.

\section{Acknowledgements}

We thank Dr D J Reynolds and $\operatorname{Dr} \mathrm{S} M$ Sheerin for the serum albumin and haematology measurements respectively, and $\operatorname{Dr}$ B S Shine for helpful advice.

\section{References}

1 Anderson MD, Collins G, Davis G, Bivins BA (1985) Malnutrition and length of stay-a relationship? Henry Ford Hosp Med J 33: 190-193.

2 Robinson G, Goldstein M, Levine GM (1987) Impact of nutritional status on DRG length of stay. J Parent Ent Nutr 11: 49-51.

3 Peiffer SC, Blust P, Leyson JFJ (1981) Nutritional assessment of the spinal cord injured patient. Perspectives in Practice 78: 501-505.

4 Newmark SR, Sublett D, Black J, Geller R (1981) Nutritional assessment in a rehabilitation unit. Arch Phys Med Rehabil 62: 279-282.

5 Laven GT, Huang C-T, DeVivo MJ, Stover SL, Kuhlemeier KV, Fine PR (1989) Nutritional status during the acute stage of spinal cord injury. Arch Phys Med Rehabil 70: 277-282.

6 Kaufman HH, Rowlands BJ, Stein DK, Kopaniky DR, Gildenberg PL (1985) General metabolism in patients with acute paraplegia and quadriplegia. Neurosurgery 16: 309-313.

7 Glenn MB, Carfi J, Belle SE, Ahn JH, Gordon WA, Myer PA et al (1985) Serum albumin as a predictor of course and outcome on a rehabilitation service. Arch Phys Med Rehabil 66: 294-297. 
8 Editorial (1986) Indices of surgical risk. Lancet 1: 1422-1423.

9 Jeejeebhoy KN, Megiod MM (1986) Assessment of nutritional status in the oncologic patient. Nutr Cancer 66: $1077-1090$.

10 Pettigrew RA (1988) Identification and assessment of the malnourished patient. Balliere's Clin Gastroenterol 2: 729-749.

11 Vinnars E, Wernerman J (1988) Metabolism and nutrition in trauma. Upsala J Med Sci 93: 131-138.

12 Lee, BY, Agarwal N, Corcoran L, Thoden WR, del Guercio LRM (1985) Assessment of nutritional and metabolic status of paraplegics. J Rehabil Res 22: 11-17.

13 Reilly JJ, Hull SF, Albert N, Waller A, Bringardener S (1987) Economic impact of malnutrition: a model system for hospitalised patients. J Parent Ent Nutr 12: 371-376.

14 Eastham RD (1984) Clinical Haematology. 6th ed. Wright, Bristol. 